\title{
Switch to agalsidase alfa after shortage of agalsidase beta in Fabry disease: a systematic review and meta-analysis of the literature
}

\author{
Antonio Pisani, MD, PhD¹, Dario Bruzzese, MD, PhD², Massimo Sabbatini, MD, PhD¹, \\ Letizia Spinelli, MD³ ${ }^{3}$ Massimo Imbriaco, $\mathrm{MD}^{4}$ and Eleonora Riccio, MD, $\mathrm{PhD}^{5}$
}

\begin{abstract}
Background: In 2009, the agalsidase beta shortage resulted in switching to agalsidase alfa treatment for many Fabry disease patients, offering the unique opportunity to compare the effects of the two drugs. Because single studies describing effects of switching on the disease course are limited and inconclusive, we performed a systematic review and meta-analysis of existing data.
\end{abstract}

Methods: Relevant studies were identified in the PubMed, Cochrane, ISI Web, and SCOPUS databases from July 2009 to September 2015. The following parameters were analyzed: clinical events, changes in organ function or structure, disease-related symptoms, lyso-Gb3 plasma levels, and adverse effects.

Conclusions: The nine studies (217 patients) included in our systematic review showed only marginal differences in most of the evaluated parameters. Seven of these studies were included in the meta-analysis (176 patients). The pooled incidence rate of major adverse events was reported for five studies (150 patients) and was equal to 0.04 events per person-year. No significant change was observed after the shift in glomerular filtration rate, whereas left ventricular mass index, left ventricular posterior wall dimension, and ejection fraction were significantly reduced over time. Our data showed that the switch to agalsidase alfa was well tolerated and associated with stable clinical conditions.

Genet Med advance online publication 8 September 2016

Key Words: agalsidase alfa; agalsidase beta; Fabry disease; shortage; switch.
Fabry disease (FD) is an $\mathrm{X}$-linked, rare, progressive, multisystemic disorder due to lysosomal enzyme $\alpha$-galactosidase A deficiency. ${ }^{1}$ It leads to intracellular accumulation of glycosphingolipids, mainly globotriaosylceramide (Gb3), in different tissues. ${ }^{2}$ Renal failure, cardiomyopathy, and peripheral and central nervous system alterations are the main causes of morbidity and reduced life expectancy. ${ }^{3}$ Treatment options for FD patients include long-term enzyme replacement therapy in addition to supportive management. ${ }^{4}$ Two recombinant enzyme formulations for enzyme replacement therapy for FD are currently available: agalsidase alfa (Replagal, Shire) and agalsidase beta (Fabrazyme, Genzyme), which are produced using different methods and administered at different doses. ${ }^{5}$ Both preparations have been shown to be safe and effective in patients with $\mathrm{FD},{ }^{6,7}$ but there are very few data that directly compare the clinical effects of the two drugs.

In June 2009, viral contamination in the manufacturing process of Fabrazyme led to a global shortage of agalsidase beta; as a consequence, patients formerly treated with the drug in a standard dose $(1.0 \mathrm{mg} / \mathrm{kg}$ e.o.w.) either received an initial administration of a reduced dose of the enzyme $(0.3-0.5 \mathrm{mg} / \mathrm{kg}$ e.o.w.) and then switched to the standard dose of agalsidase alfa $(0.2 \mathrm{mg} / \mathrm{kg} \text { e.o.w. })^{8-10}$ or had their treatment immediately shifted to agalsidase alfa. ${ }^{8-10}$ This offered the opportunity to compare the effects of the two drugs throughout the course of the disease, although indirectly. Unfortunately, the exiguous number of patients included in each study and the relatively short follow-up period did not allow evidence-specific modifications or adverse events to occur after the shift.

Therefore, to explore these issues, we performed extensive research of the available literature with evidence reported in a systematic review and a meta-analysis of all the available data.

\section{MATERIALS AND METHODS}

\section{Data sources and study selection}

The study was designed according to the Meta-Analysis of Observational Studies in Epidemiology (MOOSE) guidelines. ${ }^{11}$ Articles published from July 2009 to September 2015 were searched in the PubMed, Cochrane, ISI Web of Science, and SCOPUS databases. We also searched conference proceedings of the most significant conferences on lysosomal storage disorders. The terms "Fabry disease," "agalsidase alfa," "Replagal,"

The first two authors contributed equally to this work.

${ }^{1}$ Nephrology Section, Department of Public Health, University Federico II of Naples, Naples, Italy; ${ }^{2}$ Statistics Section, Department of Public Health, University Federico II of Naples, Naples, Italy; ${ }^{3}$ Department of Advanced Biomedical Sciences, Federico II University, Naples, Italy; ${ }^{4}$ Department of Radiology, Federico II University of Naples, Naples, Italy; ${ }^{5}$ Department of Nephrology, Second University of Naples, Naples, Italy. Correspondence: Antonio Pisani (antonio.pisani@libero.it) 
"agalsidase beta," or "Fabrazyme" were searched and crossed with "switch," "switching," or "shortage."

We included all studies regardless of publication status (published, conference proceedings, or unpublished), study duration, and language.

Two reviewers independently inspected each reference identified by the search and applied inclusion criteria. For possibly relevant articles or in case of disagreement, we obtained and independently inspected the full article.

\section{Eligibility criteria}

Studies were included if they reported on outcomes after switching from agalsidase beta to agalsidase alfa in FD patients, and they were excluded if any of the following criteria applied: (i) duplicate publication; (ii) case report, case series, comments, expert opinions, editorials, or commentaries; (iii) studies that analyzed the same sample of a preexisting study; or (iv) lack of data on outcomes after switching therapy.

\section{Data extraction}

Two reviewers independently extracted data from the included studies and any discrepancy was resolved by consensus. We contacted all investigators of included studies for missing data.

\section{Outcome measures}

To quantify the clinical outcome, the following FD-related progression parameters were analyzed in the qualitative analysis.

Clinical events. These included (i) death; (ii) cardiac events such as symptomatic arrhythmia requiring implantation of an implantable cardioverter-defibrillator or pacemaker, myocardial infarction, coronary artery bypass graft, or percutaneous transluminal coronary angioplasty; (iii) renal events such as progression of chronic kidney disease (CKD) to stage 5, i.e., estimated glomerular filtration rate (eGFR) $<15 \mathrm{ml} / \mathrm{min}$ per $1.73 \mathrm{~m}^{2}$ (with decrease of eGFR $\geq 30 \%$ ) necessitating kidney transplantation or dialysis; and (iv) neurologic events such as stroke or transient ischemic attack.

Changes in organ function or structure. Modifications of organ function were investigated at the cardiac, renal, and neurologic levels. Cardiac changes were evaluated by echocardiographic data (such as thickness of cardiac structures, left ventricular volume, measures of systolic and diastolic function, heart rate), electrocardiographic data, and magnetic resonance imaging parameters. Renal function was evaluated by changes in eGFR and in urine albumin-to-creatinine ratio (UACr) in spot samples. Neurologic changes were determined on the basis of clinical examination, interview regarding stroke or stroke-like symptoms, and quantitative sensory testing for cold detection threshold assessment.

Changes in FD-related symptoms and questionnaires. These symptoms included gastrointestinal pain; diarrhea; hypohidrosis or anhidrosis; tinnitus; acroparesthesia, chronic pain, and pain crises, as assessed by the Graded Chronic Pain Score and the Neuropathic Pain Symptom Inventory score, the Brief Pain Inventory questionnaire, ${ }^{12}$ and the McGill pain questionnaire, ${ }^{13}$ fatigue; the Mainz Severity Score Index (MSSI); ${ }^{14}$ and quality of life (QoL) as determined by the Short Form $36\left(\right.$ SF-36) ${ }^{15}$ or Euro-Qol dimensions. ${ }^{16}$

Adverse effects. The adverse effects (AES) considered were dyspnea, hypertension, gastrointestinal symptoms, rigors, temperature change sensation, fever, headache, rhinitis, flushing, pruritus, and antibody formation.

\section{Quantitative data analysis and synthesis}

The parameters evaluated in the quantitative analysis were eGFR, UACr, left ventricular mass index (LVMI), left ventricular posterior wall dimension (LVPWD), and ejection fraction (EF). Statistical analyses were performed according to the statistical guidelines developed by The Cochrane Collaboration and published in the Cochrane Handbook for Systematic Reviews of Interventions. ${ }^{17}$ For each study, the mean variation with $95 \%$ confidence interval (CI) was computed. When not directly available, the standard error of the mean difference was estimated from the reported variances of baseline and final values or from the available individual patient data (G3Data Graph Analyzer, version 1.5.3). Overall estimates were computed with a fixed-effect model or random-effects model depending on whether the amount of heterogeneity was low (i.e., $\left.\mathrm{I}^{2}<50 \%\right)$ or moderate to high ( $\mathrm{I}^{2}$ $>50 \%$ ). Random-effects model meta-regression analyses were also performed to examine the effect of the duration of the agalsidase alfa regimen after the switch on the outcomes. Incidence of major adverse effects was meta-analyzed by using a randomeffects Poisson regression, as described by Bagos. ${ }^{18}$ All analyses were performed with $R$ statistical version 2.15.0. The significance level for the overall estimates of effect was set at $P<0.05$.

\section{Units}

Values for the following parameters are given throughout in the first unit listed and may be converted for the second unit by multiplying by the conversion factor provided: $\mathrm{UACr} \mathrm{mg} / \mathrm{g}$ to $\mathrm{g} / \mathrm{g}, 0.001 ; \mathrm{mg} / \mathrm{mmol}$ to $\mathrm{g} / \mathrm{g}, 0.0084$.

\section{RESULTS}

The literature search identified 86 publications regarding treatment switching in FD patients; of them, only 56 were potentially eligible publications because the remaining 30 represented duplicate records. Following the exclusion reasons reported in Figure 1, only 9 studies fulfilled our search strategy and were included in the qualitative synthesis; ${ }^{19-27}$ however, two of these lacked detailed statistical methods $\mathrm{s}^{26,27}$ and were excluded from the meta-analysis that, accordingly, consisted of 7 studies. ${ }^{19-25}$ The study by Lenders ${ }^{22}$ includes the results of the same patients described by Weidemann ${ }^{28}$ after a longer follow-up (2 years vs. 1 year); accordingly, the data by Weidemann were not considered in the quantitative analysis, with the exclusion of those data not reported by Lenders. 


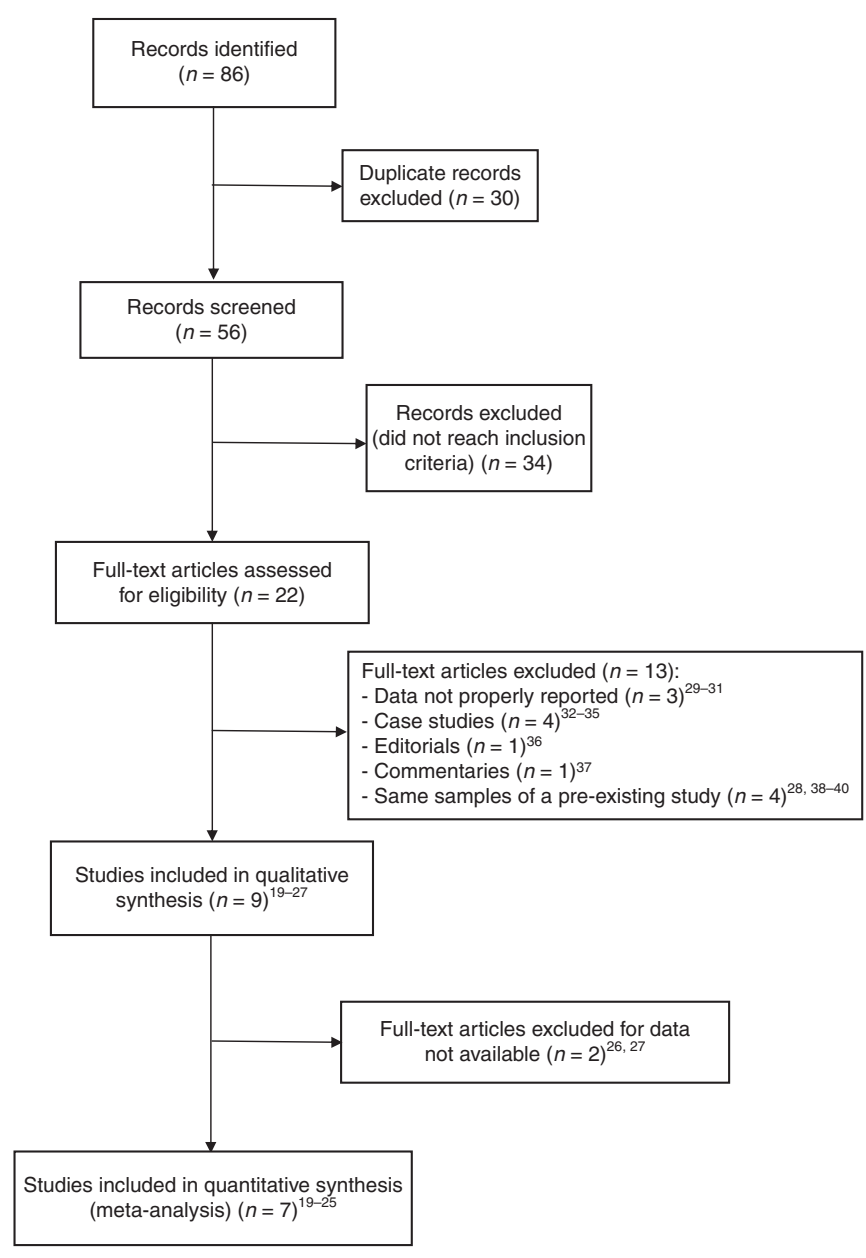

Figure 1 PRISMA flow diagram of the search strategy.

\section{Qualitative synthesis}

The studies of qualitative analysis reported data regarding an overall population of $217 \mathrm{FD}$ patients. Table 1 summarizes the main characteristics of the included publications. Wide variability was found between the studies regarding the characteristics of participants, length of treatment period, and measured outcomes. Most of data from the study by $\mathrm{Smid}^{20}$ were not considered in such analyses, although they were reported by the authors because they represent a pool of data from patients treated with halved doses of agalsidase beta for 6 months before the shift to agalsidase alfa.

Clinical events. Six studies explored this outcome..$^{20-23,25,27}$ Eight clinical events occurred in 154 patients after the switch (5.2\%) during a median follow-up period of 24 months.

Of these, two were reported by Goker-Alpan $(2.8 \%$ of his patients) and included one death caused by cardiac arrest and a case of transient ischemic attack. ${ }^{25} \mathrm{~A}$ case of atrial fibrillation was described by Tsuboi (9.1\% of patients), ${ }^{21}$ and two additional, although not specified, events were observed by Smid (10\% of patients). ${ }^{20}$ Lenders observed three clinical events (8.1\% of patients): one case of end-stage renal disease with hemodialysis, one transient ischemic attack/stroke, and the implantation of a pacemaker/cardioverter-defibrillator. ${ }^{22}$ In the last two publications, ${ }^{23,27}$ no clinical event was reported.

Changes in organ function or structure. These changes were categorized as cardiac, renal, and neurologic.

Seven publications reported cardiac modifications after the switch $(19-23,25,26 ; n=158$ patients); data from Smid's study ${ }^{20}$ could not be evaluated because the results were reported as pooled data that included patients immediately switched to agalsidase alfa at time of $\beta$ shortage and those remaining on halved agalsidase beta doses. Data were quite heterogeneous; indeed, septal wall thickness significantly improved in one study, ${ }^{21}$ was unchanged in another, ${ }^{22}$ and was significantly worsened after switch in another. ${ }^{26}$ Similarly, a significant improvement in LVMI occurred in two studies, ${ }^{19,21}$ but no change was reported in the remaining ones. ${ }^{23,25,26}$ No difference was described in midwall fractional shortening, ${ }^{25} \mathrm{EF}^{22,23}$ and LVPWD. ${ }^{23,26}$ Tsuboi observed a significant improvement in LVPWD $(P=0.00236)$ and worsening in EF $(P=0.0340) 3$ years after the switch. ${ }^{21}$ Notably, no change in electrocardiogram parameters was reported in two studies. ${ }^{21,22}$

Renal function after the switch was evaluated in all studies; ${ }^{19-27}$ however, as for cardiac changes, data found by $\mathrm{Smid}^{20}$ were not considered. No change was reported in seven studies for UACr ${ }^{19,21-26}$ because data from Reidt's study ${ }^{27}$ were not available, and no change was reported for eGFR. ${ }^{19,21,23-27}$ Interestingly, Lenders ${ }^{22}$ evaluated eGFR using three different CKD-EPI formulas based on creatinine and cystatine C: (i) creatinine-based (eGFR creat $_{\text {eat }}$; (ii) cystatine C-based (eGFR ${ }_{\text {cys }}$ ); and (iii) creatinine/cystatine C-based (eGFR creat-cys $_{\text {s }}$ ) Although $\mathrm{eGFR}_{\text {creat }}$ remained stable during the 2-year follow-up period, both $\mathrm{eGFR}_{\text {cys }}$ and $\mathrm{eGFR}_{\text {creat-cys }}$ values decreased significantly over time after the shift $(P<0.01)$.

Neurologic changes were reported in only one study, ${ }^{22}$ but the authors did not describe significant changes in any of the evaluations performed.

Change in FD-related symptoms. Four studies reported on MSSI. $19,21,22,26$ No change was described after switching in two studies (21, 26; $n=37$ patients). Lin reported stability of the disease at 12 months, with mild improvements in cardiac or renal scores for three out of nine patients and a slight deterioration in general score for one patient. ${ }^{19}$ Worsened MSSI, conversely, was observed by Lenders ( $P=0.001, n=37$ patients). ${ }^{22}$

Four studies reported on pain..$^{20-23}$ Also, for pain, the effects of switch could not be evaluated in the study by Smid $;{ }^{20}$ the remaining three studies showed no difference in pain severity after the switch. ${ }^{21-23}$ Moreover, Lenders reported that patients with switched treatment had less frequent pain attacks compared to patients using agalsidase beta, although they developed greater gastrointestinal pain. ${ }^{22}$

Three studies reported changes in QoL, ${ }^{20,21,23}$ with no significant modifications in the different scales of QoL before and after switching. ${ }^{21,23}$ 


\begin{tabular}{|c|c|c|c|c|c|}
\hline Reference & $N$ & $\begin{array}{l}\text { Duration of } \\
\text { preshortage } \\
\text { ERT period }\end{array}$ & $\begin{array}{l}\text { Duration of } \\
\text { follow-up } \\
\text { period }\end{array}$ & Study outcomes & Main results \\
\hline Tsuboi ${ }^{21}$ & 11 & $\begin{array}{l}47.8 \pm 19.4 \\
\text { months" }\end{array}$ & 3 years & $\begin{array}{l}\text { - Changes in clinical events; } \\
\text { - Cardiac status: LVMI, LVPWd, SWT, EF, ECG, BNP; } \\
\text { - Renal function: eGFR, UACr; } \\
\text { - pain; QoL; MSSI; } \\
\text { - Lyso-Gb3; antibody analysis }\end{array}$ & $\begin{array}{l}\text { - No change in all evaluated parameters; } \\
\text { - Improvement in LVMI, SWT, LVPWd, EF }\end{array}$ \\
\hline Pisani²3 & 10 & $>48$ months & 20 months & $\begin{array}{l}\text { - Changes in clinical events; } \\
\text { - Cardiac status: LVMI, LVPWd, EF, ECG; } \\
\text { - Renal function: eGFR, UACr; } \\
\text { - Patient health status; QoL; pain }\end{array}$ & - No change in all evaluated parameters \\
\hline Wakabayashi24 & 18 & $\begin{array}{l}36.3 \pm 17.1 \\
\text { months }\end{array}$ & 2 years & - Renal function: sCr, SUN, eGFR, UACr & - No change in all evaluated parameters \\
\hline Goker-Alpan ${ }^{25}$ & 71 & $\begin{array}{c}4.6 \text { years } \\
(0.3-12.2)^{*}\end{array}$ & 2 years & $\begin{array}{l}\text { - Changes in clinical events; } \\
\text { - Cardiac status: LVMI, MFS; } \\
\text { - Renal function: eGFR, UACR }\end{array}$ & - No change in all evaluated parameters \\
\hline
\end{tabular}

BNP, brain natriuretic peptide; BP, blood pressure; CKD, chronic kidney disease; ECG, electrocardiogram; EF, ejection fraction; eGFR, estimated glomerular filtration rate; FD, Fabry disease; LVMI, left ventricular mass index; LVPWD, left ventricular posterior wall diameter; MSSI, Mainz Severity Score Index; QoL, quality of life; sCr, serum creatinine; SUN, serum urea nitrogen; SWT; septal wall thickness; UACr, urine albumin to creatinine ratio.

Data are expressed as means \pm SD $(\#)$ or as median and interquartile range $\left(^{*}\right)$.

Only Lenders reported that changes in other symptoms such as diarrhea, tinnitus, and ability to sweat were not detected after the switch. ${ }^{22}$

Change in lyso-Gb3 plasma concentration. Four studies reported on this outcome. ${ }^{19-21,25}$ In two publications, plasma lysoGb3 levels gradually decreased during treatment with agalsidase beta, and no significant difference was observed after the switch. ${ }^{19,21}$ Similarly, Goker-Alpan did not report changes after the switch. ${ }^{25}$ By contrast, Smid et al. ${ }^{20}$ reported an increase in lyso-Gb3 in male patients switched to agalsidase alfa after 1 year of shortage (median increase: $8.1 \mathrm{nmol} / \mathrm{l}$; range: $2.5-29.2$ ); however, all patients with switched treatment and an available lyso-Gb3 value underwent a 6 -month dose reduction to $25 \%$ of the initial dose of agalsidase beta before the shift.

Adverse effects. Five studies ( $n=121$ patients) reported on this outcome. ${ }^{19-21,23,25} \mathrm{~A}$ total of 30 patients experienced at least one AE after the switch ( $24.8 \%$ of patients). Pisani ${ }^{23}$ reported infusion adverse reactions in six patients (54.5\% of patients), which consisted of rigors, fever, nausea, headache, vomiting, rhinitis, flushing, or pruritus. Most infusion adverse reactions were assessed as mild and decreased over time. No AE was observed by Tsuboi $^{21}$ after switching; however, one male patient who developed antibodies against agalsidase beta and experienced worsening of allergic rhinitis during the infusions recovered after the switch. Smid ${ }^{20}$ reported one possible/ probable infusion adverse reaction consisting of infusionrelated vomiting, asthenia, chills, and increased blood pressure that resolved without sequelae. In the study by $\operatorname{Lin},{ }^{19}$ two patients presented hypersensitivity reactions after the switch (22.2\% of patients), which were represented by breath shortness, pruritus, and fever in the first patient and an itchy, red plaque on the limbs and diarrhea in the second patient. However, in both patients similar AE had already occurred during agalsidase beta treatment. Finally, Goker-Alpan ${ }^{25}$ reported 
"serious," although unspecified, AE in 21 patients after the shift (29.6\% of his cohort). However, it should be considered that such percentages did not differ from those observed in naive patients $(37.9 \%)$ or patients previously treated with agalsidase$\alpha(27.3 \%)$, respectively.

The pooled incidence rate of major adverse events was reported in five studies ${ }^{20,21,23,25,28}$ including 150 patients and was equal to 0.04 events per person-year ( $95 \% \mathrm{CI}: 0.01$ to 0.19 ).

\section{Meta-analysis results}

After the screening, seven studies were included in the final meta-analysis $^{19-25}$ ( $n=176$ patients), although some of them did not report information about all the investigated outcomes. This analysis also included the data of LVMI, LVPWD, EF, and UACr from Weidemann's study, ${ }^{28}$ which were not reported by Lenders $^{22}$ in his 2-year follow-up of the same patients.

All the studies included in the meta-analysis reported the values of eGFR. ${ }^{19-25}$ Analysis of these data showed that the switch determined no significant modifications in GFR at the end of follow-up (mean change from baseline: $-0.52 \mathrm{ml} /$ $\min / 1.73 \mathrm{~m}^{2} ; 95 \% \mathrm{CI}:-3.22$ to $+2.19 ; P$ value from the random-effects model $=0.708)$ (Figure 2$)$. We performed a sensitivity analysis and removed from that analysis the study of Tsuboi, ${ }^{21}$ which showed an outlying effect size. When this study was excluded there was no more evidence of statistical heterogeneity $\left(\mathrm{I}^{2}=0 \%\right)$, but the random-effects model yielded comparable results (mean change from baseline: $-1.37 \mathrm{ml} /$ $\mathrm{min} / 1.73 \mathrm{~m}^{2}$; $95 \% \mathrm{CI}:-3.53$ to $+0.80 ; P$ value from the random-effects model $=0.216$ ). Duration of follow-up did not affect the variations in eGFR, as evidenced by meta-regression analysis $(P=0.356)$.

Six studies ( $n=159$ patients) reported information on LVMI. ${ }^{19-}$ ${ }^{21,23,25,28}$ A random-effects model $\left(\mathrm{I}^{2}=60.6 \%, \mathrm{Q}=10.16, P=0.038\right)$ showed a significant reduction in LVMI after the switch (mean change from baseline: $-4.2 \mathrm{~g} / \mathrm{m}^{2}$; $95 \% \mathrm{CI}:-8.66$ to $-0.25 ; P<$ 0.034) (Figure 3 ). No effect of duration of follow-up on LVMI variation was observed by meta-regression $(P=0.623)$.

Table 2 reported the summary estimates of mean changes from baseline for LVPWD, EF, and UACr in three studies including 59 patients. ${ }^{21,23,28}$ A significant decrease was observed in LVPWD (mean change from baseline: $-0.69 \mathrm{~mm}, P<0.001$ ) and also in $\mathrm{EF}$ (mean change from baseline: $-3.51 \%, P=0.023$ )

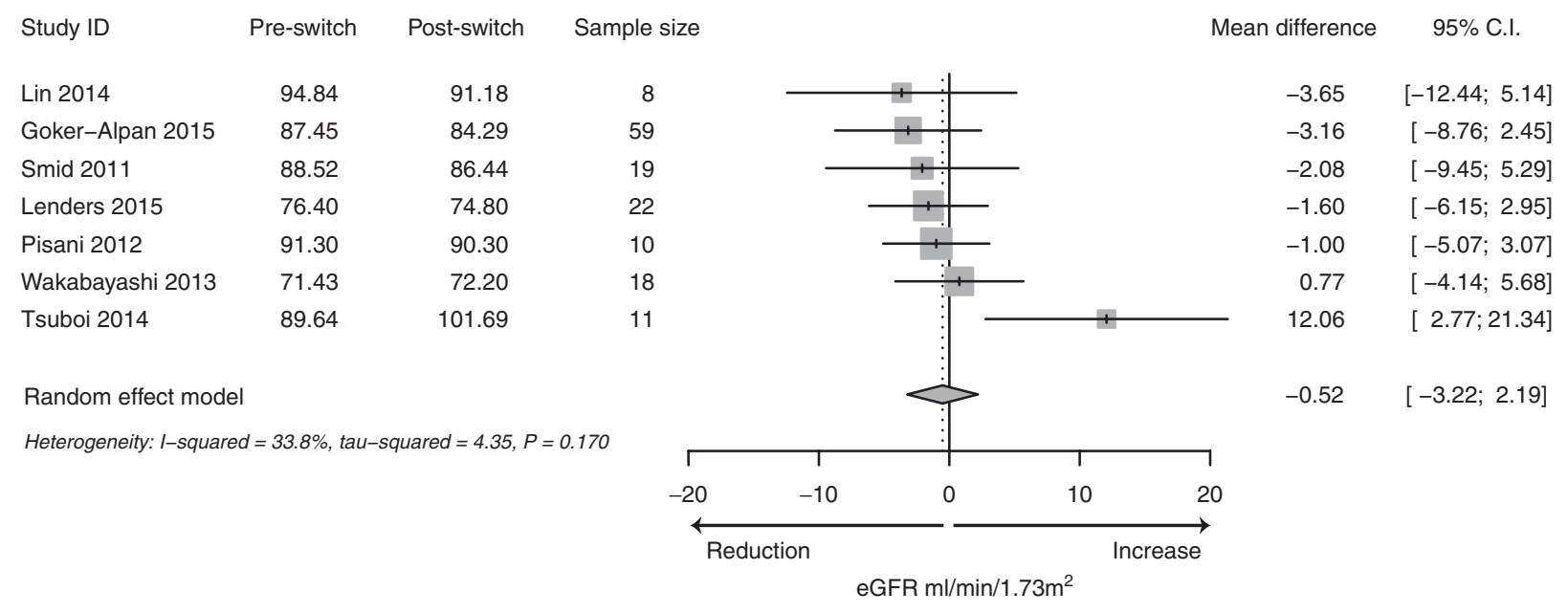

Figure 2 Changes from baseline in eGFR values in patients switched from agalsidase beta to agalsidase alfa.

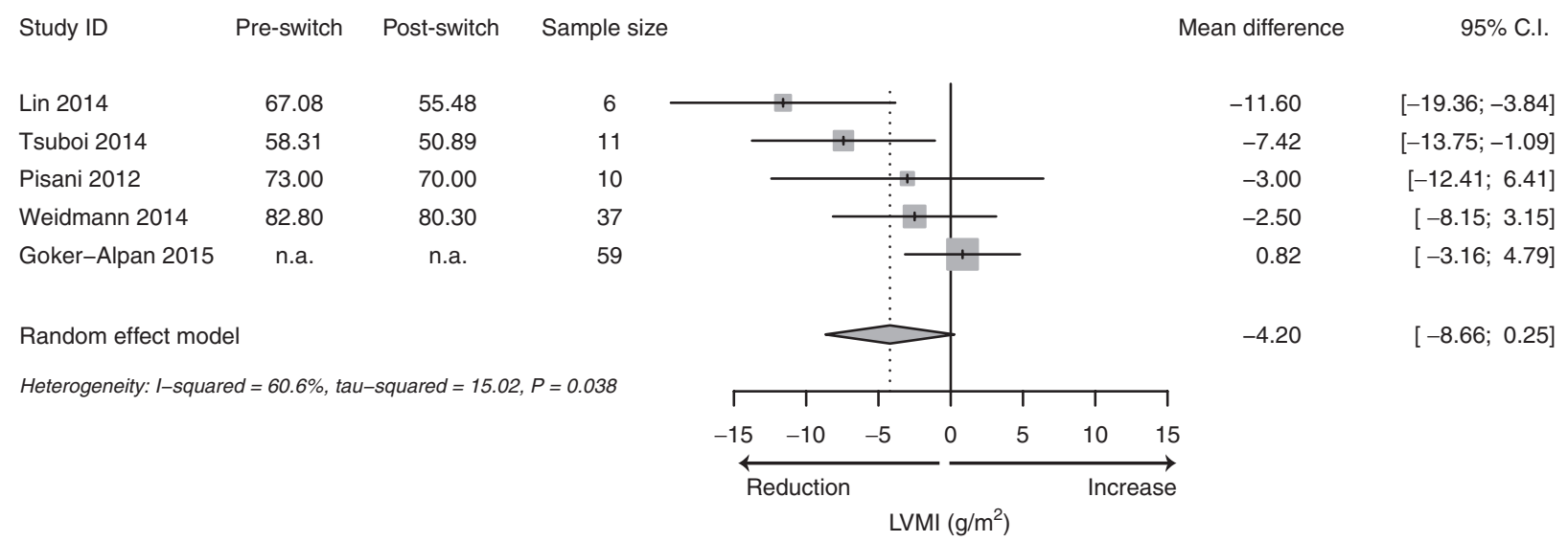

Figure 3 Changes from baseline in LVMI in patients switched from agalsidase beta to agalsidase alfa*. *Data from the Goker-Aplan study were obtained by graphical extraction (GraphClick software) that allowed determination of the changes from baseline (and $95 \% \mathrm{Cl}$ ) in absence of raw data (n.a.). 
Table 2 Mean change from baseline of LVPWD, EF, and $\operatorname{UACr}(n=59$ patients).

\begin{tabular}{|c|c|c|c|c|}
\hline $\begin{array}{l}\text { Outcome } \\
\text { variable }\end{array}$ & $\begin{array}{c}\text { Mean change } \\
(95 \% \mathrm{Cl})\end{array}$ & $P$ value & $\begin{array}{l}\text { Heterogeneity } \\
\left(1^{2} \text { index, \%) }\right.\end{array}$ & $\begin{array}{c}Q \text { statistic } \\
P \text { value }\end{array}$ \\
\hline $\begin{array}{l}\text { LVPWD } \\
(\mathrm{mm})\end{array}$ & $-0.69(-1.02$ to -0.36$)$ & $<0.001$ & 0 & 0.414 \\
\hline $\mathrm{EF}(\%)$ & $-3.51(-6.55 ;-0.48)$ & 0.023 & 43.4 & 0.171 \\
\hline $\begin{array}{l}\text { UACr } \\
(\mathrm{g} / \mathrm{gCr})\end{array}$ & $-7.67(-49.66$ to 34.31$)$ & 0.721 & 0 & 0.517 \\
\hline
\end{tabular}

EF, ejection fraction; LVPWD, left ventricular posterior wall dimension; UACr, urinary albumin to creatinine ratio.

after the switch, but the UACr ratio did not change, in agreement with eGFR data.

\section{DISCUSSION}

Following the agalsidase beta shortage in 2009, a considerable number of patients with FD were switched to agalsidase alfa. This allowed the opportunity to compare, although indirectly, the two drugs through the evaluation of clinical modifications or adverse events that occurred after the switch. In fact, although the effects of both treatments have been extensively described in the literature, the possibilities to compare the two drugs were limited and inconclusive. This is not surprising because the studies on FD are observational and include few nonrandomized patients with short follow-up times, as typically occurs in "rare" diseases, and their data often do not allow for evidence changes in organ function or in disease-related symptoms. Therefore, any conclusion about the effects of the switch from individual studies is inconsistent.

To overcome these limits, we performed a meta-analysis that included a complete review of the literature and whose primary objective was to evaluate the safety and tolerability of agalsidase alfa in FD patients previously treated with agalsidase beta.

The data of the present study support the conclusion that agalsidase alfa does not worsen renal and cardiac function or FD-related symptoms, at least in the short term.

As the first step, we performed a qualitative evaluation of the typical manifestations of FD occurring with both drugs (Table 1) and involving nine studies and 218 patients, which clearly suggested that there was no apparent change after the shift at the renal or cardiac level, with just a few exceptions.

Interestingly, the incidence of clinical events was definitely low with agalsidase alfa treatment $(0.04$ events per personyear; $95 \%$ CI: 0.01 to 0.19$)$. Moreover, two events described by Smid occurred in patients previously treated with halved doses of agalsidase beta before the shift, ${ }^{20}$ so it is not clear whether their onset should be ascribed to such reduction or to a presumed lower efficacy of agalsidase alfa. This latter study, ${ }^{20}$ however, deserves attention because it compares the incidence of clinical events/year/person from the start of agalsidase beta treatment during the halved doses at the time of shortage and after the shift to agalsidase- $\alpha$. Notably, Smid showed that there was no statistical difference in the number of clinical events that occurred with agalsidase alfa $(n=2)$ compared to those observed during the reduced agalsidase beta dose $(n=5)(P=$
0.11 ); there was no statistical difference in the number of clinical events that occurred with full-dose agalsidase beta with a similar follow-up period (males: $P=0.25$; females: $P=0.14$ ).

The low number of clinical events, described in all the studies, is even more important considering that in FD their incidence increases with time following the natural progression of the disease that occurs even despite therapy. Moreover, the EMA report ${ }^{9}$ warned about a possible accelerated course of FD and increased incidence of AE following agalsidase- $\beta$ dose reductions or switch to agalsidase- $\alpha$. This statement created deep concern in patients and physicians and led to an overestimation of events and AEs (the so-called "switch effect").

Similar conclusions may be drawn for renal and cardiac functions. All studies examining renal function strongly agreed regarding the stability of eGFR and UACr after the shift, with one exception, ${ }^{22}$ and showed a significant decrease over time in eGFR ( $P<0.05$ vs. baseline) in both groups with reduced agalsidase- $\beta$ doses and agalsidase alfa treatments when renal function was calculated by two formulas measuring cystatine and cystatine plus creatinine. ${ }^{22}$ However, it is noteworthy that when eGFR was measured by creatinine (CKD-EPI), no significant change was observed compared to baseline in any group during the follow-up.

Data regarding cardiac function demonstrated clear stability in most of the examined parameters; in particular, all the studies showed that LVMI did not change or even modestly improved after the shift. ${ }^{19,21}$ West $^{26}$ reported a negative impact of $\alpha$-agalsidase on septal wall thickness (6.4\% increase, $P<0.03$ vs. baseline); however, it was not confirmed by two further studies. ${ }^{19,21}$ All the remaining data on cardiac parameters, like LVPWD, midwall fractional shortening, and EF, were quite uniform and showed steadiness over time. Also, the negative data reported by Tsuboi, who described significant worsening in $\mathrm{EF}$ after the shift $(P=0.0340)$, must be correctly interpreted because EF largely remained within the range of normality after the shift (mean: $65.8 \%) .^{21}$

Although it is questionable to evaluate clinical deterioration on the basis of subjective criteria, most of the questionnaires describing FD symptoms remain largely subjective. Nevertheless, despite the worries induced by the EMA report, MSSI score, referred pain, QoL, and other FD-related symptoms remained stable in most studies, with the only exception being the study by Lenders, who described worsening of MSSI score counterbalanced by a reduction of pain attacks frequencies. $^{22}$ Therefore, for subjective symptoms, it seems that no peculiar difference exists between the two treatments.

An objective measure of disease progression, conversely, is represented by the changes in plasma lyso-Gb3 levels. Only Smid showed a significant increase in lyso-Gb3 levels in male patients whose treatment was switched to agalsidase alfa at the end of follow-up (1.3 years) compared to baseline..$^{20}$ As observed for clinical events, however, the authors emphasize that all patients with switched treatment and an available lyso-Gb3 value were previously treated with reduced doses of agalsidase beta. Therefore, this recurrence in disease activity (with no correlation with clinical events during the follow-up period) could 
be attributed to the reduced agalsidase beta dose, thus supporting the previous observations that the critical determinant of the biochemical response is not the type of enzyme preparation, but rather its dose. Indeed, no change in this parameter could be detected in the other three studies in which the shift was performed with equivalent doses of agalsidase alfa, ${ }^{19,21,25}$ despite considerably longer follow-up periods.

The data of the meta-analysis, involving 176 patients from seven studies, overlap the observations of the "qualitative" analysis of the single studies, thus showing that there is no evidence that treatment with agalsidase alfa negatively influences the progression of FD, at least in the short term.

Meta-analysis, in fact, confirmed the stability of renal function (evaluated by eGFR or UACr) over time and the steadiness of cardiac parameters despite the small improvements in LVMI and LVPWD and the slight worsening in EF; however, although the differences after the shift in both of these latter data reached statistical significance, they seem to have little (if any) clinical impact on heart function but strengthen the concept that agalsidase alfa is safe at the cardiac level.

The limits of the present study are the same as those reported in the single studies, including the low number of patients, the short observation period (only 11 patients had a 3-year follow-up!), the heterogeneity of the study population, and, in particular, the low number of clinical events that demonstrate the slow progression of the disease with enzyme replacement therapy, thus reflecting the intrinsic nature of rare genetic diseases. We therefore cannot exclude the possibility that the incidence of clinical events or symptoms may increase over a longer observation period, nor can we understand the degree to which the incidence of adverse events is conditioned by the baseline conditions of the single patients or by the duration of the disease and patients' age (both increased during the follow-up).

In conclusion, the findings of the present study confirm that the switch to agalsidase alfa is safe in terms of side effects. Although the data from the meta-analysis show that the use of agalsidase alfa is associated with steady levels of GFR and minor, although significant, improvements in cardiac function, the heterogeneity of data and, most importantly, the short follow-up period do not enable us to determine whether clinical stability and efficacy are maintained in the long term. New data collected after prolonged follow-up periods are warranted to definitively demonstrate the comparable effects of the two drugs.

\section{DISCLOSURE}

A.P. is a consultant for Shire and Genzyme. He received grants and travel support from Shire, Genzyme, and Amicus. The other authors declare no conflict of interest.

\section{REFERENCES}

1. Desnick R, lonnou Y, Eng C. Fabry disease: alpha galactosidase A deficiency. In: The Metabolic and Molecular Bases of Inherited Disease. Scriver C, Beaudet A, Sly W, Valle D (eds.). McGraw-Hill: New York, 1995:2741-2784.

2. Faggiano A, Pisani A, Milone F, et al. Endocrine dysfunction in patients with Fabry disease. J Clin Endocrinol Metab 2006;91:4319-4325.
3. Desnick RJ, Brady R, Barranger J, et al. Fabry disease, an under-recognized multisystemic disorder: expert recommendations for diagnosis, management, and enzyme replacement therapy. Ann Intern Med 2003;138:338-346.

4. Pisani A, Sabbatini M, Duro G, et al. Antiproteinuric effect of add-on Paricalcitol in Fabry disease patients: a prospective observational study. Nephrol Dial Transplant, 2014; 0: 1-6.

5. Pisani A, Visciano B, Imbriaco M, et al. The kidney in Fabry's disease. Clin Genet 2014;86:301-309.

6. Pisani A, Visciano B, Roux GD, et al. Enzyme replacement therapy in patients with Fabry disease: state of the art and review of the literature. Mol Genet Metab 2012;107:267-275.

7. Lidove $\mathrm{O}$, West ML, Pintos-Morell G, et al. Effects of enzyme replacement therapy in Fabry disease-a comprehensive review of the medical literature. Genet Med 2010;12:668-679.

8. Europeans Medicines Agency. Assessment report on the shortage of Fabrazyme. Overview of shortage period: Spontaneous reports from June 2009 through 15 September 2010 and Registry data from June 2009 through 05 August 2010. 19 November 2010. http://www.ema.europa.eu/docs/en_GB/ document_library/Other/2010/11MC500099241.pdf.

9. European Medicines Agency. European Medicines Agency reviews treatment recommendations for Fabrazyme. 22 October 2010. http://www.ema.europa. eu/docs/en GB/document library/Press release/2010/10/WC500098370.pdf.

10. Linthorst GE, Germain DP, Hollak CE, et al.; European Medicines Agency. Expert opinion on temporary treatment recommendations for Fabry disease during the shortage of enzyme replacement therapy (ERT). Mol Genet Metab 2011;102:99-102.

11. Stroup DF, Berlin JA, Morton SC, et al. Meta-analysis of observational studies in epidemiology: a proposal for reporting. Meta-analysis Of Observational Studies in Epidemiology (MOOSE) group. JAMA 2000;283:2008-2012.

12. Tan G, Jensen MP, Thornby Jl, Shanti BF. Validation of the brief pain inventory for chronic nonmalignant pain. J Pain 2004;5:133-137.

13. Melzack R. The short-form McGill Pain Questionnaire. Pain 1987;30:191-197.

14. Whybra C, Kampmann C, Krummenauer F, et al. The Mainz Severity Score Index: a new instrument for quantifying the Anderson-Fabry disease phenotype, and the response of patients to enzyme replacement therapy. Clin Genet 2004;65:299-307.

15. Ware JE. SF-36 Health Survey: Manual and Interpretation Guide. The Health Institute, New England Medical Centre: Boston, MA, 1997.

16. The EuroQoL Group. EuroQoL-a new facility for the measurement of health related quality of life. Health Policy, 1990; 16: 199-208.

17. Higgins JPT, Green S. Cochrane Handbook for Systematic Reviews of Interventions Version 5.1.0 [updated March 2011]. The Cochrane Collaboration, 2011. http://www.cochrane-handbook.org.

18. Bagos PG, Nikolopoulos GK. Mixed-effects Poisson regression models for metaanalysis of follow-up studies with constant or varying durations. Int J Biostat 2009;5:1557-4679.

19. Lin HY, Huang YH, Liao HC, et al. Clinical observations on enzyme replacement therapy in patients with Fabry disease and the switch from agalsidase beta to agalsidase alfa. J Chin Med Assoc 2014;77:190-197.

20. Smid BE, Rombach SM, Aerts JM, et al. Consequences of a global enzyme shortage of agalsidase beta in adult Dutch Fabry patients. Orphanet J Rare Dis 2011;6:69.

21. Tsuboi K, Yamamoto H. Clinical course of patients with Fabry disease who were switched from agalsidase- $\beta$ to agalsidase- $\alpha$. Genet Med 2014;16:766-772.

22. Lenders M, Canaan-Kühl S, Krämer J, et al. Patients with Fabry disease after enzyme replacement therapy dose reduction and switch-2-year follow-up. J Am Soc Nephrol 2016;27:952-962.

23. Pisani A, Spinelli $L$, Visciano $B$, et al. Effects of switching from agalsidase Beta to agalsidase alfa in 10 patients with anderson-fabry disease. JIMD Rep 2013;9:41-48.

24. Wakabayashi T, Sakuma M, Morita A, et al. The effect of switching treatment from agalsidase- $\beta$ to agalsidase- $\alpha$ on renal function in 18 adults with Fabry disease. 12th International Congress of Inborn Errors of Metabolism (ICIEM), Barcelona, 3-6 September 2013.

25. Goker-Alpan O, Nedd K, Suma P, et al. Effect and tolerability of Agalsidase alfa in patients with Fabry disease who were treatment naïve or formerly treated with Agalsidase beta or Agalsidase alfa. JIMD Rep 2015;23:7-15.

26. West M, Bichet DG, Casey R, et al. The Canadian Fabry Initiative. Lysosomal Storage Disease Network WORLD Symposium, San Diego, CA, 8-10 February 2012. 
27. Reidt S, Namdar M, Serra A, et al. Disease progress in patients with Morbus Fabry after switching from agalsidase beta to agalsidase alpha. Intern Med J 2014;44:205-207.

28. Weidemann F, Krämer J, Duning T, et al. Patients with Fabry disease after enzyme replacement therapy dose reduction versus treatment switch. J Am Soc Nephrol 2014;25:837-849.

29. Sirrs S, Clarke JT, Bichet DG, et al. Baseline characteristics of patients enrolled in the Canadian Fabry Disease Initiative. Mol Genet Metab 2010;99:367-373.

30. Thompson L, Bleakley C, Hallows L. Experience of switching enzyme replacement therapy (ERT) products in patients with Anderson Fabry disease: a specialist nurse perspective. J Inherit Metab Dis 2010;33(suppl 1):S153. Abstract 492-P.

31. Sirrs SM, Bichet DG, Casey R, et al.; CFDI investigators. Outcomes of patients treated through the Canadian Fabry disease initiative. Mol Genet Metab 2014;111:499-506

32. Hallows $L$, Waldek $S$. Our experiences of switching patients from Fabrazyme to Replagal at Salford Royal NHS Foundation Trust. J Inherit Metab Dis 2010;33(suppl 1):S1-S197. Abstract 494-P.

33. Tanaka A, Takeda T, Hoshina T, Fukai K, Yamano T. Enzyme replacement therapy in a patient with Fabry disease and the development of lgE antibodies against agalsidase beta but not agalsidase alpha. J Inherit Metab Dis 2010;33(suppl 3):S249-S252.
34. Eyskens FJM, De Boeck K. Switch from Fabrazyme to Replagal in the treatment of patients with Fabry disease: the need for an individual approach. J Inherit Metab Dis 2011;34(suppl 3):S228. Abstract P-444.

35. Tsuboi K, Yamamoto H, Somura F, Goto H. Successful management of enzyme replacement therapy in related fabry disease patients with severe adverse events by switching from agalsidase beta (Fabrazyme®) to agalsidase alfa (Replagal®). JIMD Rep 2015;15:105-111.

36. Warnock DG, Mauer M. Fabry disease: dose matters. J Am Soc Nephrol 2014;25:653-655.

37. Pisani A, Riccio E, Sabbatini M. Agalsidase alfa and agalsidase beta in the treatment of Fabry disease: does the dose really matter? Genet Med 2015;17:21-23.

38. Tsuboi K, Yamamoto H. Clinical observation of patients with Fabry disease after switching from agalsidase beta (Fabrazyme) to agalsidase alfa (Replagal). Genet Med 2012;14:779-786.

39. Pisani A, Visciano B, Capuano I, et al. A comparison of the therapeutic efficacy of two different enzyme treatments in Fabry's disease: from evidence in the literature to personal experience. G Ital Nefol 2013;30.

40. Riccio E, Pisani A. [The switch of enzyme therapy in Fabry disease]. G Ital Nefrol $2014 ; 31$ 